\title{
Correction to: Linear Time-Invariant Systems, Behaviors and Modules
}

\section{Correction to:}

U. Oberst et al., Linear Time-Invariant Systems, Behaviors and Modules, Differential-Algebraic Equations Forum, https://doi.org/10.1007/978-3-030-43936-1

The original version of the book was published with few errors in Chaps. 8 and 10 . These have been corrected in the updated version. 